\title{
Wavelet filter techniques for segmenting retinal blood vessels
}

\author{
Abdulsamad Al-Marghilnai ${ }^{1, *}$, Romany F. Mansour ${ }^{2}$ \\ ${ }^{1}$ College of Computer Science and Information, Northen Border University, Arar, Saudi Arabia \\ 2 Department of Computer Science, Faculty of Science, Northern Border University, Arar, Saudi Arabia
}

\section{ARTICLE INFO}

\section{Article history:}

Received 29 May 2017

Received in revised form

2 August 2017

Accepted 5 August 2017

\section{Keywords:}

Retinal image

Wavelet filter

Retinal blood vessels

\begin{abstract}
A B S T R A C T
Retinal fundus image is generally used to examine the diabetic retinopathy symptoms, by analysing blood vessel segmentation and also access the pathologies of the eye. Retinal blood vessel details can be mined from retinal fundus images through image processing. Processing involves three stages, Pre-processing, Segmentation, and Post-processing. Among the different segmentation algorithms existing, the wavelet filter method has been shown to be highly advantageous in distinguishing blood vessels effectively. Under this method, the objects in noisy background can be segmented and hence sort out the image from the background in a finer way. Retinal images obtained from retinal databases like DRIVE and STARE can be analysed using the wavelet filter algorithm. Herein wavelet filter method will be evaluated for efficient segmentation of the retinal blood vessels using retinal images obtained from databases.
\end{abstract}

(C) 2017 The Authors. Published by IASE. This is an open access article under the CC BY-NC-ND license (http://creativecommons.org/licenses/by-nc-nd/4.0/).

\section{Introduction}

Difference in blood vessel diameters takes place as part of the independent control of blood flow in healthy person and can be according to the altered stages in the pulse cycle (Knudtson et al., 2004), while continued change in the diameter may also point towards the existence of some pathology (Patton et al., 2006). Retinal vessel narrowing can be related to incidents of coronary heart disease in women occurrence of stroke and diabetes in men and women (Wong et al., 2002). Narrowing of the retinal vessels can also be related with hypertension, ageing, inflammation and other processes.

Investigation and recognition of fundamental alteration on the retinal blood vessel are very significant to identify and sense retinal disease such as diabetic hypertension, retinopathy, arteriosclerosis and age-related macular degeneration (AMD) (You et al., 2011). Premature symptoms of such sicknesses are pointed out by features of retinal blood vessel like length, diameter, angle, and tortuosity (Martinez-Perez et al., 2002). Retinal blood vessel is the only part of the blood circulation that could be directly observed and studied in detail (Mansour et al., 2013). Retinal imaging is additionally utilized to decide on retinal

\footnotetext{
* Corresponding Author.

Email Address: srd.nbu@gmail.com (A. Al-Marghilnai) https://doi.org/10.21833/ijaas.2017.09.022

2313-626X/C 2017 The Authors. Published by IASE.

This is an open access article under the CC BY-NC-ND license

(http://creativecommons.org/licenses/by-nc-nd/4.0/)
}

normality and to identify or supervise retinal irregularities and defects like modifications in thickness and tortuosity of the retinal blood vessel are pointers for increased risk levels of diabetic retinopathy (Owen et al., 2011). Thus retinal irregularities and defects are mainly prominent in diabetic retinopathy (DR) which is a vascular impediment of diabetes mellitus and is a major reason of vision loss among diabetic patients. For patients suffering from diabetes, it is important that diabetic retinopathy be monitored and detected at the earliest and avoid vision loss (AOA, 2014).

Identifying malformations like venous looping or beadings is important for before time action as they are in general warning of probable sight-threatening retinopathy. To make use of these valuable descriptions of retinal blood vessels, it is imperative to evaluate their position and shapes precisely (Sharma and Kothari, 2017). In order to analyse the retinal blood vessels, retinal fundus images are collected and processed to analyse blood vessel segmentations (Mansour, 2016). The processing of these imagines includes pre-processing, segmentation and post-segmentation.

During pre-processing, noise on the retina image is removed, while during segmentation aberrations in blood vessel can be detected using various methods, such as watershed algorithm, probabilistic filters, wavelet filter algorithm, Gabor filter (Li et al., 2006). Subsequently, blood vessel abnormalities previously undiscovered in retinal imaging can be detected using these segmentation procedures (Aras 
et al., 2016). Fig. 1 presents various features of the generic DR image.

Programmed segmentation decreases the time required for physical labelling and analysis of retinal images. Therefore a dependable vessel segmentation process like wavelet filter method will be important for premature detection of changes in the retina due to diseases like diabetes. The objective of the paper is to evaluate the advantages of Wavelet Filter method for segmenting retinal blood vessels during image signal processing.

\section{Literature review}

\subsection{Overview of the segmentation process}

Every retinal fundus image undergoes processing before the blood vessels can be analysed, which are divided into pre-processing stage and segmentation stage. Moreover, several databases are also maintained of such retinal fundus images, for the purpose of comparative analysis and research for practitioners and researchers.

Table 1: Retinal images databases

\begin{tabular}{|c|c|c|}
\hline S. No. & DATABASE & DESCRIPTION \\
\hline 1 & DRIVE Database & $\begin{array}{l}\text { - Publically available database of fundus images collected from } 435 \text { people in Netherland, age } \\
\text { group of } 31-86 \text { gathered via diabetic retinopathy screening program. } \\
\text { - } 40 \text { images in the database, of which } 20 \text { are training images and } 20 \text { test images stored in 24- } \\
\text { bits color, size of } 768 \times 584 \text { pixels (Chhabra and Bhushan, 2014). }\end{array}$ \\
\hline 2 & STARE Database & $\begin{array}{l}\text { - Facilitates blood vessel segmentation. } \\
\text { - Stores } 20 \text { images of size } 700 \times 605 \text { pixels with } 8 \text { bit per colour channel (Zhao et al., 2014). } \\
\text { - Facilitates comparative studies on retinal images using automatic segmentation algorithms. }\end{array}$ \\
\hline 3 & HRF & $\begin{array}{l}\text { - Contains } 45 \text { images divided into three sets of fundus images: } 15 \text { of glaucomatous, } 15 \text { of } \\
\text { healthy and } 15 \text { of diabetic retinopathy subjects (Odstrčilík, 2014). }\end{array}$ \\
\hline 4 & ARIA Online & $\begin{array}{l}\text { - Database consists of three groups: } 59 \text { images of infected retinas under diabetes group, } 61 \\
\text { images of control group and } 92 \text { images of macular degeneration group based on age (Fraz } \\
\text { et al., 2012). }\end{array}$ \\
\hline 5 & Image RET & $\begin{array}{l}\text { - Database divided into two sub-databases, DIARETDB0 and DIARETDB1 (Fraz et al., 2012) } \\
\text { - DIARETDB0- } 130 \text { retina images, of which } 20 \text { are normal images and remaining } 110 \text { images } \\
\text { include images with a variety of symptoms of diabetic retinopathy. } \\
\text { - DIARETDB1- } 89 \text { retina images of which } 5 \text { images are healthy images and rest are with } \\
\text { symptoms of diabetic retinopathy }\end{array}$ \\
\hline 6 & Messidor & $\begin{array}{l}\text { - Largest database of } 1200 \text { retina images. } \\
\text { - Images collected from } 3 \text { ophthalmology departments with the resolutions of } 1440 \times 960 \text {, } \\
2240 \times 1488 \text {, and } 2304 \times 1536 \text { pixels respectively } \\
\text { - TIFF format images }\end{array}$ \\
\hline
\end{tabular}

\subsection{Pre-processing procedures}

Pre-processing includes a group of procedures that are used to remove error or sound from the retina images and to augment the contrast value on fundus images and further increases the accuracy of the image via correcting the brightness and normalisation (Aras et al., 2016). Pre-processing procedures involve Brightness Level Equalization which uses green channel as feature descriptor since green channel produces highest contrast on retina image thereby distinguishing the features (Saleh et al., 2011). Further image normalisation is used for colour alteration, brightness rectification and contrast augmentation (Kumar et al., 2012). Hue channel escalates the contrast of blood vessels (Relan et al., 2014) and Homomorphic filtering is used for image improvement and rectification by regularizing the brightness of the entire image

\subsection{Retinal image database}

In medical science, an important instrument for the dependable assessment and comparison of algorithms related to image processing is a database that consists of a particular group of medical images of high-quality (Kauppi et al., 2006).

Such images serve as base for diabetic retinopathy analysis and can be extracted from retinal image database. There are many retinal databases available namely digital retinal image for vessel extraction, STARE database, HRF, ARIA and are presented in Table 1 (Aras et al., 2016).

These databases hold extensive collections of retinal images that can help researchers in analysing and comparing retinal images using different segmentation algorithms.

These databases typical consist of retinal images of healthy as well as patients suffering from diabetic retinopathy. Besides these, many other databases are also available, such as ROC Microaneurysm Set, CHASE_DBI Database and Retinal Vessel Tortuosity Dataset (Aras et al., 2016). 
analysis and detection of blood vessels. The postprocessing process eliminates the redundant region and makes the vessel as major point of concentration. Correctness of segmentation can also be enhanced via post processing procedures (Li et al., 2012) after which the salt and pepper noise is removed using a $3 \times 3$ median filter. Isolated pixel can be removed using length filtering and morphological operators (Patankar et al., 2013). Further a twostage process was proposed by Zhao et al. (2014) for final segmentation result to obtain a clear blood vessel for analysis from the retinal image. The first step involves combining is various retinal vessel segmentation results using the region growing and level set method. The step two involves eliminating fake detection of the standalone vessel pixels.

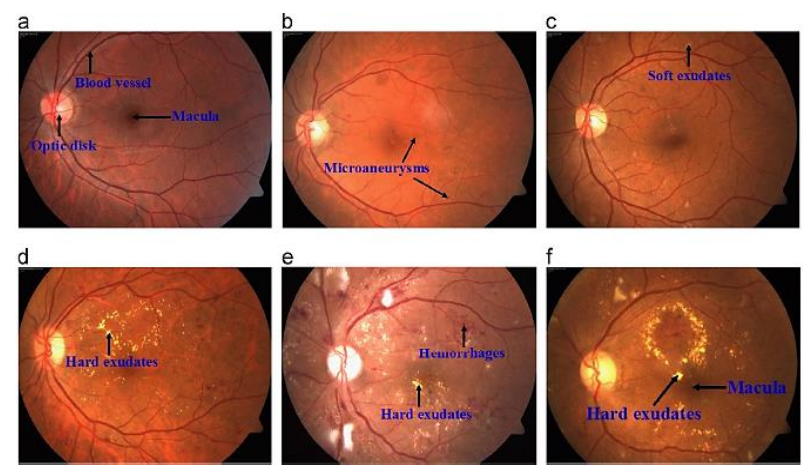

Fig. 1: Varied features of the diabetic retinopathy image (Mansour, 2017)

\section{Wavelet filter method for segmentation of retinal blood vessel: Overview}

Retinal images are images that are obtained by creating photographs of backside of eye and are also called fundus or ocular images (Jadhav and Patil, 2015). Retinal blood vessels measurement and detection can be used to categorize the disease' rigorousness. Segmentation procedure involves sorting out background and foreground regions in the image. The foreground regions involve the clear retina area containing the vessels while the background includes the regions outside the boundaries of the retina area which is the noise or not required area (Mansour, 2012). Retinal blood vessels segmentation contains quantifiable alteration in diameter, branching angles and length, effect of disease (Lee, 2006). Therefore, dependable method of blood vessel segmentation and extraction is important for recognition and categorization of alteration. Mainstream methods used for vessel segmentation are based on watershed algorithm, probabilistic filters, wavelet filter algorithm, Gabor filter and such methods (Li et al., 2006) out of which in this study wavelet filter method will be used for segmenting retinal blood vessels.

A wavelet is a wave-like alternation with amplitude that starts at zero, increases, and then decreases back to zero that is of limited duration instead of stating from minus infinity to plus infinity as the case of continuous sinusoidal wave. Wavelets are used to build an image pyramid which symbolizes the entropy levels for each frequency which is used to segment objects in noisy images based on their frequency reaction in a variety of frequency bands, sorting them out from the background and from other objects. They are designed to display precise possessions that make them helpful for signal and wave processing. Thus, a wavelet filter consists of finite number of discrete points (Gavlasová et al., 2006). Joshi and Karule, (2012) gave a process to divide the retinal vessel by means of two-dimensional administered categorization and Morlet wavelet. This research creates segmentations by classifying each pixel of image as non-vessel or vessel based on the feature vector (FV) in pixel. Feature vector are concerned with strength of pixels and uninterrupted two dimensional Morlet wavelet transforms were well thought-out. In a research conducted by Akram et al. (2009) a two-dimensional advancement of Gabor wavelet was developed for programmed segmentation of blood vessels (BV) which is effectual for treatment of images in the vessels under dissimilar conditions with rational dependability and correctness for medical analysis. Shabbir et al. (2013) evaluated and estimated the automated process for blood vessels improvement and segmentation in RI. It was established that Gabor wavelet and global threshold method is better for the improvement and segmentation of vessel. Table 2 show comparison between some techniques for segmentation of retinal blood vessel.

The Figs. 2, 3, and 4 describe the Daubechies wavelet filter for different lengths. These are family of orthogonal wavelets defining a discrete wavelet transform. Further, the formula applicable in any wavelet filter algorithm is related to the basic function of the wavelet. For any $L$ function $f(x)$, the continuous wavelet transformation is defined as a function of two variables.

$\operatorname{CWT}_{f}(a, b)=\left(f, \psi_{a, b}\right)=\int f(x) \overline{\psi_{a, b}(x)} d x$.

Here the dilation and translation parameters, $a$ and $b$, respectively, vary continuously over $\mathrm{R} \backslash\{0\} \times$ R.
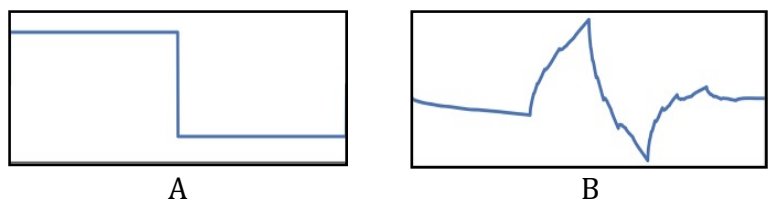

Fig. 2: Daubechies Wavelet External Phase filters. (A) Filter with length 2. (B) Filter with length 4
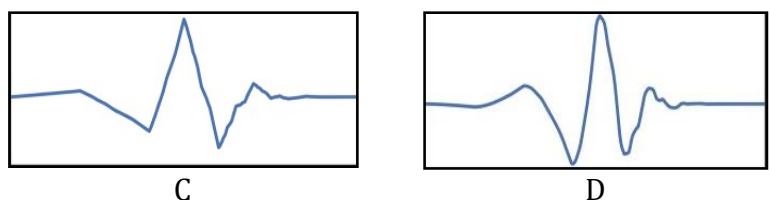

Fig. 3: (C) Filter with length 6. (D) Filter with length 8 


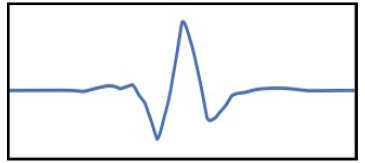

$\mathrm{E}$

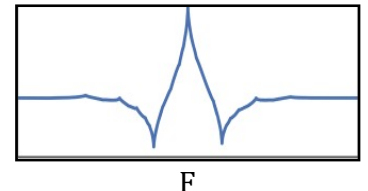

$\mathrm{F}$
Fig. 4: (E) Daubechies Least Asymmetric filters with length 8. (F) Coiflet filter with length 6

Table 2: Retinal segmentation of retinal blood vessel

Author
Singh and Singh
(2014)
Han et al. (2014)
Cinsdikici and
Aydin (2009)

\section{Discussions and conclusion}

Segmentation of blood vessel serves as the basis for developing retinal screening systems since it helps in identification and categorization of features of retina. The screening of irregularities of vessel can be done using an effectual and proficient approach for automated blood vessels segmentation in retinal images. An appropriate method of image processing could give a hand in recognizing defects in retinal vessels from image of fundus. Mainstream methods used for vessel segmentation include multiple algorithms as discussed, of which wavelet filter method will be adopted. The retinal fundus images obtained from database undergo pre-processing to remove error or sound from the retina images and to augment the contrast value on fundus images, followed by segmentation for detection of the blood vessel using wavelet filter. This method includes limited oscillation waveform thus obtaining the retina blood vessel. Hence the present research evaluates the efficacy of using wavelet filters for segmentation of the retinal blood vessels by removing high frequency noise.

\section{References}

Akram MU, Atzaz A, Aneeque SF, and Khan SA (2009). Blood vessel enhancement and segmentation using wavelet transform. In the International Conference on Digital Image Processing, IEEE, Bangkok, Thailand: 34-38. https://doi.org/10.1109/ICDIP.2009.70

Akram MU, Jamal I, Tariq A, and Imtiaz J (2012). Automated segmentation of blood vessels for detection of proliferative diabetic retinopathy. In the IEEE-EMBS International Conference on Biomedical and Health Informatics (BHI), IEEE, Hong Kong, China: 232-235. https://doi.org/10.1109/BHI. 2012.6211553

AOA (2014). Diabetes eye care of the patient with diabetes mellitus. American Optometric Association, Virginia, USA.

Aras RA, Lestari T, Nugroho HA, and Ardiyanto I (2016). Segmentation of retinal blood vessels for detection of diabetic retinopathy: A review. Communications in Science and Technology, 1(1): 33-41.

Chhabra S and Bhushan B (2014). Supervised pixel classification into arteries and veins of retinal images. In the Innovative Applications of Computational Intelligence on Power, Energy and Controls with their impact on Humanity, IEEE, Ghaziabad, India: 59-62. https://doi.org/10.1109/CIPECH.2014.7019098

Cinsdikici MG and Aydın D (2009). Detection of blood vessels in ophthalmoscope images using MF/ant (matched filter/ant colony) algorithm. Computer Methods and Programs in Biomedicine, 96(2): 85-95

Fraz MM, Remagnino P, Hoppe A, Uyyanonvara B, Rudnicka AR, Owen CG, and Barman SA (2012). Blood vessel segmentation methodologies in retinal images-a survey. Computer Methods and Programs in Biomedicine, 108(1): 407-433.

Gavlasová, A., Procházka, A., \& Mudrová, M. (2006). Wavelet based image segmentation. In the $14^{\text {th }}$ Annual Conference Technical Computing, Prague, Czech Republic. Available online at: http://www.humusoft.cz/ftp/www/papers/tcp06/gavlasova. $\mathrm{pdf}$

Han Z, Yin Y, Meng X, Yang G, Yan X (2014) Blood vessel segmentati on in pathological retinal image. In the IEEE International Conference on Data Mining Workshop, IEEE, Shenzhen, China: 960-967. https://doi.org/10.1109/ICDMW. 2014.16

Jadhav A and Patil BP (2015). Classification of diabetes retina images using blood vessel area. International Journal on Cybernetics and Informatics, 4(2): 251-257.

Joshi S and Karule PT (2012). Retinal blood vessel segmentation. International Journal of Engineering and Innovative Technology (IJEIT), 1(3): 175-178.

Kauppi T, Kalesnykiene V, Kamarainen JK, Lensu L, Sorri I, Uusitalo H, and Pietilä J (2006). DIARETDB0: Evaluation database and methodology for diabetic retinopathy algorithms. Machine Vision and Pattern Recognition Research Group, Lappeenranta University of Technology, Finland, Available online at: http://www.it.lut.fi/project/imageret/ diaretdb0/doc/diaretdb0_techreport_v_1_1.pdf

Knudtson MD, Klein BEK, Klein R, Wong TY, Hubbard LD, Lee KE, and Bulla CP (2004). Variation associated with measurement of retinal vessel diameters at different points in the pulse cycle. British Journal of Ophthalmology, 88(1): 57-61.

Kumar A, Gaur AK, and Srivastava M (2012). Segment based technique for detecting exudate from retinal fundus image. Procedia Technology, 6: 1-9.

Lee THH (2006). Wavelet Analysis for Image Processing. Institute of Communication Engineering, National Taiwan University, Taipei, Taiwan. Available online at: https://pdfs.semanticscholar.org/aff9/9f7e32890bb6843255 e2bd6c0ff0092dfeab.pdf

Li Q, You J, and Zhang D (2012). Vessel segmentation and width estimation in retinal images using multiscale production of matched filter responses. Expert Systems with Applications, 39(9): 7600-7610. 
Li Q, You J, Zhang L, and Bhattacharya P (2006). A multiscale approach to retinal vessel segmentation using Gabor filters and scale multiplication. In the IEEE International Conference on Systems, Man and Cybernetics (SMC'06), IEEE, Taipei, Taiwan: 4: 3521-3527. https://doi.org/10.1109/ICSMC.2006. 384665

Mansour R (2017). Evolutionary computing enriched computer aided diagnosis system for diabetic retinopathy: A survey. IEEE Reviews in Biomedical Engineering, IEEE Engineering in Medicine and Biology Society IEEE Consumer Electronics Society [Technical Co-Sponsor], PP(99): 1-1. https://doi.org/10.1109/RBME.2017.2705064

Mansour RF (2012). Using genetic algorithm for identification of diabetic retinal exudates in digital color images. Journal of Intelligent Learning Systems and Applications, 4(03): 188198.

Mansour RF (2016). Iris recognition using gauss laplace filter. American Journal of Applied Sciences, 13(9): 962-968.

Mansour RF, Abdelrahim EM, and Al-Johani AS (2013). Identification of diabetic retinal exudates in digital color images using support vector machine. Journal of Intelligent Learning Systems and Applications, 5(03): 135-142.

Martinez-Perez ME, Highes AD, Stanton AV, Thorn SA, Chapman N, Bharath AA, and Parker KH (2002). Retinal vascular tree morphology: A semi-automatic quantification. IEEE Transactions on Biomedical Engineering, 49(8): 912-917.

Mustafa WA, Yazid H, and Yaacob SB (2014). A review: Comparison between different type of filtering methods on the contrast variation retinal images. In the IEEE International Conference on Control System, Computing and Engineering, IEEE, Batu Ferringhi, Malaysia: 542-546. https://doi.org/10.1109/ICCSCE.2014.7072777

Odstrčilík PIJ (2014). Analysis of retinal image data to support glaucoma diagnosis. Available online at: https://core.ac.uk/ download/pdf/30311092.pdf

Owen CG, Rudnicka AR, Nightingale CM, Mullen R, Barman SA, Sattar N, and Whincup PH (2011). Retinal arteriolar tortuosity and cardiovascular risk factors in a multi-ethnic population study of 10-year-old children; the child heart and health study in England (CHASE). Arteriosclerosis, Thrombosis, and Vascular Biology, 31(8): 1933-1938.

Patankar SS, Mone AR, and Kulkarni JV (2013). Gradient features and optimal thresholding for retinal blood vessel segmentation. In the IEEE International Conference on Computational Intelligence and Computing Research, IEEE, Enathi, India: 1-5. https://doi.org/10.1109/ICCIC.2013. 6724116
Patton N, Aslam TM, MacGillivray T, Deary IJ, Dhillon B, Eikelboom RH, and Constable IJ (2006). Retinal image analysis: concepts, applications and potential. Progress in Retinal and Eye Research, 25(1): 99-127.

Relan D, MacGillivray T, Ballerini L, and Trucco E (2014). Automatic retinal vessel classification using a least squaresupport vector machine in VAMPIRE. In the $36^{\text {th }}$ Annual International Conference of the IEEE Engineering in Medicine and Biology Society, IEEE, Chicago, USA: 142-145. https://doi.org/10.1109/EMBC.2014.6943549

Saleh MD, Eswaran C, and Mueen A (2011). An automated blood vessel segmentation algorithm using histogram equalization and automatic threshold selection. Journal of Digital Imaging, 24(4): 564-572.

Shabbir S, Tariq A, and Akram MU (2013). A comparison and evaluation of computerized methods for blood vessel enhancement and segmentation in retinal images. International Journal of Future Computer and Communication, 2(6): 600-603.

Sharma N and Kothari P (2017). Study on segmentation of retinal blood vessels using wavelet filter methodology. International Journal of Innovative Research in Computer and Communication Engineering, 5(1): 1059-1065.

Singh D and Singh B (2014). A new morphology based approach for blood vessel segmentation in retinal images. In the Annual IEEE India Conference, IEEE, Pune, India: 1-6. https://doi.org/10.1109/INDICON.2014.7030686

Sun K, Chen Z, Jiang S, and Wang Y (2011). Morphological multiscale enhancement, fuzzy filter and watershed for vascular tree extraction in angiogram. Journal of Medical Systems, 35(5): 811-824.

Wong TY, Klein R, Sharrett AR, Schmidt MI, Pankow JS, and Couper DJ (2002). Retinal arteriolar narrowing and risk of diabetes mellitus in middle-aged persons. Jama, 287(19): 2528-2533.

You S, Bas E, Erdogmus D, and Kalpathy-Cramer J (2011). Principal curved based retinal vessel segmentation towards diagnosis of retinal diseases. In the First IEEE International Conference on Healthcare Informatics, Imaging and Systems Biology, IEEE, San Jose, USA: 331-337. https://doi.org/10.1109/HISB.2011.39

Zaki SKM, Zulkifley MA, and Nazari A (2014). Tracing of retinal blood vessels through edge information. In the IEEE International Conference on Control System, Computing and Engineering, IEEE, Batu Ferringhi, Malaysia: 13-17. https://doi.org/10.1109/ICCSCE.2014.7072681

Zhao YQ, Wang XH, Wang XF, and Shih FY (2014). Retinal vessels segmentation based on level set and region growing. Pattern Recognition, 47(7): 2437-2446. 